\title{
The evolution of contrail microphysics in the vortex phase
}

\author{
Simon Unterstrasser*1, KLAUS Gierens ${ }^{1}$ and Peter Spichtinger ${ }^{2}$ \\ ${ }^{1}$ Deutsches Zentrum für Luft- und Raumfahrt, Institut für Physik der Atmosphäre, Oberpfaffenhofen, Germany \\ ${ }^{2}$ Institute for Atmospheric and Climate Science, ETH Zurich, Switzerland
}

(Manuscript received November 7, 2006; in revised form October 18, 2007; accepted December 17, 2007)

\begin{abstract}
We investigate the evolution of contrails during the vortex phase using numerical simulations. Emphasis is placed on microphysical properties and on the vertical distribution of ice mass and number concentration at the end of the vortex phase. Instead of using a 3D model which would be preferable but computationally too costly, we use a 2D model equipped with a special tool for controlling vortex decay. We conduct a great number of sensitivity studies for one aircraft type. It turns out that atmospheric parameters, namely supersaturation, temperature, stability and turbulence level have the biggest impact on the number of ice crystals and on the ice mass that survives until vortex breakup and that therefore makes up the persistent contrail in supersaturated air. The initial ice crystal number density and its distribution in the vortex, are of minor importance.
\end{abstract}

\begin{abstract}
Zusammenfassung
Die Entwicklung von Kondensstreifen während der Wirbelphase wird mit Hilfe numerischer Simulationen studiert. Besonderes Augenmerk wird auf mikrophysikalische Eigenschaften und die vertikale Verteilung des Eises (Masse and Anzahlkonzentration) am Ende der Wirbelphase gelegt. Anstelle eines 3D-Modells, was wünschenswert, aber zu rechenzeitintensiv wäre, verwenden wir ein 2D-Modell, das mit einem Spezialwerkzeug zum Steuern des Wirbelzerfalls ausgerüstet wird. Damit kann eine große Anzahl von Sensitivitätsstudien für einen festgelegten Flugzeugtyp durchgeführt werden. Es stellt sich heraus, dass atmosphärische Parameter, nämlich Übersättigung, Temperatur, Stabilität und Turbulenzniveau den größten Einfluss auf Anzahldichte und Massenkonzentration der Eiskristalle haben, die bis zum Wirbelzerfall überleben und die folglich den persistenten Kondensstreifen in übersättigter Luft bilden. Die anfängliche Anzahl der Eiskristalle und deren Verteilung in den Wirbeln sind hingegen von untergeordneter Bedeutung.
\end{abstract}

\section{Introduction}

Of all aviation effects on the radiative forcing of the atmosphere that due to persistent contrails that evolve into contrail cirrus is potentially the largest (IPCC, 1999; SAUSEN et al., 2005; MANNSTEIN and SCHUMANN, 2005; S TUBER et al., 2006). However, the degree of scientific understanding of contrail cirrus' share of aviations radiative forcing is still judged so low that authors of the IPPC special report and of the TRADEOFF ${ }^{1}$ study (SAUSEN et al., 2005) didn't dare to ascibe a best estimate to this effect. Indeed, it is very difficult to set up a climatology of contrail cirrus, a prerequisite for any attempt to quantify its radiative forcing. While it is relatively easy to detect line-shaped contrails in satellite images automatically (MANNSTEIN et al., 1999), no method is currently known to distinguish contrail cirrus from natural cirrus in satellite data after a contrail has lost its characteristic line shape. Numerical studies on the contrail-to-cirrus transition are rare (GIERENS and

\footnotetext{
*Corresponding author: Simon Unterstrasser, Deutsches Zentrum für Luft- und Raumfahrt, Institut für Physik der Atmosphäre, Oberpfaffenhofen, Germany 82234 Wessling, Germany, e-mail: simon.unterstrasser@dlr.de

${ }^{1}$ an EU FP5 research project (2000-2003)
}

JENSEN, 1998; JENSEN et al., 1998), so that the vast variety of possible evolution pathways of aging contrails has not yet been fathomed in the least.

In order to prepare for more numerical studies of the contrail-to-cirrus transition it is necessary to provide initial conditions. It is generally not a good idea to start a simulation of a contrail cirrus at the jet exit because this would require to resolve small scales of the order $1 \mathrm{~m}$ (time step of a fraction of a second) in a domain of perhaps several kilometers and for a simulation period of several hours. A better strategy seems to be to use conditions at the end of the vortex phase as initial conditions for simulations of the contrail-to-cirrus transition. Vortex phase lidar observations and numerical studies by SUSSMANN and GIERENS $(1999,2001)$ show that the early evolution of a contrail depends sensitively on the ambient relative humidity and on the type of aircraft. Other strong sensitivities are conceivable, yet have not been studied systematically so far. Thus we think, before starting simulations of the later contrail evolution with more or less ad hoc estimates of initial conditions, we should first strive for a more complete overview for possible contrail evolution during the vortex phase, providing a better justified set of initial conditions. 
Therefore, in this paper we will carry out numerical simulations of contrail evolution during the vortex phase. Our main purpose is an investigation of the factors that mainly determine how much ice mass and how many ice crystals survive the vortex phase and how they are spatially distributed after vortex decay. To facilitate a systematic investigation we need a fast numerical model that enables to run a large number of simulations in reasonable time. Hence, we perform 2D simulations with a special method for a proper representation of vortex decay, described in detail below in sections 2 and 3. Results are presented in section 4 and discussed in section 5 . Finally, conclusions are drawn in section 6 .

\section{Model setup}

The present study covers only the vortex phase of contrail evolution, that is, the simulations start at a plume age of about $20 \mathrm{~s}$ when the vortex roll-up is completed and the temperature of the exhaust jets has relaxed to ambient values (GERZ and EHRET, 1997). The formation of contrail ice particles takes only a few seconds and is completed at the beginning of the vortex phase (see Fig. 2 in STRÖM and GIERENS, 2002). Ice nucleation therefore is not needed to be simulated. The large-eddy simulations have been carried out with the non-hydrostatic anelastic model EuLag (SMOLARKiEwiCZ and MARgolin, 1997). The tool allows to switch between a semi-lagragian advection scheme and an Eulerian approach. We opted for the Eulerian mode which employs the built-in positive definite advection algorithm MPDATA (SMOLARKIEWICZ and MARGOLIN, 1998). MPDATA is an iterative advancement of the fundamental upstream differencing method minimizing its dispersiveness. The horizontal direction $x$ is along wingspan and $z$ is the vertical coordinate. The computation of eddy viscosity uses Smagorinsky's closure.

For the calculations of the ice microphysics we used a recently developed parameterisation (SPICHTINGER and GIERENS, 2008) of bulk microphysics based on a consistent two moment scheme (i.e. prognostic equations for ice crystal mass concentration and number density). The following microphysical processes from the parameterisation are used for this study: deposition growth and sublimation of ice crystals (KOENIG, 1971; GIERENS, 1996), and sedimentation. In contrast to other models, for instance the classical approach by KESSLER (1969), all ice crystals start to fall relative to the ambient air once they are formed, i.e. there are no different classes for cloud ice and sedimenting ice. Crystal aggregation is neglected because it is unimportant for young contrails (GIERENS, 1996). For the ice crystal mass distribution we prescribe a lognormal distribution with a fixed geometrical width $\sigma_{m}=3.246$ but variable modal mass $m_{0}$ :

$$
f(m)=\frac{N}{\sqrt{2 \pi} \ln \sigma_{m} m} \cdot \exp \left[-\frac{1}{2}\left(\frac{\ln m / m_{0}}{\ln \sigma_{m}}\right)^{2}\right]
$$

The total ice crystal number concentration is $N$, the ice mass concentration $I W C$ is the first moment of the mass distribution. For the ice crystals columnar shape is assumed. Via the mass-size-relation $m=a \cdot L^{b}$ (HEYMSFIELD and IAQUINTA, 2000) the size distribution is also of lognormal type with a geometrical standard deviation of $\sigma_{L}=1.708$.

We do not use explicit ice nucleation (as e.g. homogeneous nucleation) in the vortex but we prescribe the ice crystal number density and the ice water content at the start of the simulations.

The configuration of the baseline case is: The domain has a horizontal/vertical extent of $x_{D}=256 \mathrm{~m}$ and $z_{D}=500 \mathrm{~m}$ with a $1 \mathrm{~m}$-resolution in each direction. The timestep is $\Delta t=0.02 \mathrm{~s}$ and the simulations stop after $t_{0}=160 \mathrm{~s}$. A large aircraft (Airbus A340, Boeing B747) with wing span $b_{\text {span }}=60 \mathrm{~m}$ is located at $z_{C A}=400 \mathrm{~m}$ (cruise altitude). The real height of the flight level is determined by the choice of temperature at cruise altitude (explained below). The body of the plane is in the middle of the domain $x_{H}=x_{D} / 2$. The engine exits are $20 \mathrm{~m}$ to the left/right of the body. The mass of the plane is $M=310000 \mathrm{~kg}$ and the flight speed is $U=250 \mathrm{~m} / \mathrm{s}$. As the simulations start at the beginning of the vortex phase it is assumed that the vortices have fully rolled up and each vortex can be initialized with a Hallock-Burnham profile of tangential velocity $\left(v_{\theta}\right)$ :

$$
v_{\theta}(r)=\frac{\Gamma_{0}}{2 \pi r} \frac{r^{2}}{r^{2}+r_{c}^{2}} .
$$

The critical radius is chosen to be $r_{c}=4 \mathrm{~m}$ and the initial circulation $\Gamma_{0}=650 \mathrm{~m}^{2} / \mathrm{s}$ (using $g M=\rho_{a i r} \Gamma_{0} b_{0} U$ with density of air $\left.\rho_{\text {air }}=0.4 \mathrm{~kg} / \mathrm{m}^{3}\right)$. Horizontal separation distance of the two vortex centres is $b_{0}=\frac{\pi}{4} b_{\text {span }}$. Weaker secondary vortices with opposite circulation shaping up for certain flap/wing configurations are not taken into account. The resulting maximum horizontal/vertical velocity is $13 \mathrm{~m} / \mathrm{s}$ and $15 \mathrm{~m} / \mathrm{s}$. We assume that all the water vapour emitted due to fuel burning is contained in the ice crystals. Assuming a fuel flow of $\dot{m}_{f}=3 \mathrm{~kg} / \mathrm{s}$ and given the emission index of water $E I_{\mathrm{H}_{2} \mathrm{O}}=1.25 \mathrm{~kg} / \mathrm{kg}$ the emitted water per flight path is $I W C_{0}=1.46 \cdot 10^{-2} \mathrm{~kg} / \mathrm{m}$. The ice crystals are assumed to be uniformly distributed in circles $\left(r_{p}=20 \mathrm{~m}\right)$ centred at the vortex cores. The number of ice crystals per meter of flight path is $N_{0}=3.4 \cdot 10^{12}$. Sensitivity tests investigating the effect of changing the initial concentration and distribution of the contrail ice will be presented in section 5.3. 
Since we are particularly interested in the fractions of ice number and mass that survive the vortex phase, we only consider low to moderate supersaturations up to $20 \%$. As shown later these fractions are most sensitive to relative humidity at low supersaturation. Although higher supersaturations exist in the upper troposphere, we do not consider such cases. At low supersaturations cirrus clouds usually do not form; hence we assume the atmosphere to be cloud free. The humidity field in the domain is uniform with constant $R H_{i}$.

The (ambient) turbulence is characterised in terms of eddy dissipation rate $\varepsilon=3.5 \cdot 10^{-5} \mathrm{~m}^{2} / \mathrm{s}^{3}$. Following the turbulence classification of SHARMAN (2005), this represents an atmosphere with light turbulence. The eddy dissipation rate can be transformed into the root mean square $q=\left(\overline{u^{\prime 2}}\right)^{1 / 2}$ of the turbulent velocity fluctuations using $\varepsilon=\frac{1}{8} \frac{q^{3}}{\Lambda}$. The typical length scale $\Lambda$ is chosen to be $100 \mathrm{~m}$.

The potential temperature increases at cruise altitude approximately with $3.2 \mathrm{~K} / \mathrm{km}$ and the temperature itself, $T_{C A}$, ranges from $209 \mathrm{~K}$ to $222 \mathrm{~K}$. Temperatures below the threshold $T_{S A}=225 \mathrm{~K}$ for $R H_{w}=60 \% \approx$ $R H_{i}=100 \%$ satisfy the Schmidt-Appleman criterion (SCHumanN, 1996), that is, contrails can form. A shift of flight altitude is solely effected by a change of $T_{C A}$ in the simulations. The pressure at the lower boundary is kept constant at $p_{0}=250 \mathrm{hPa}$ for all simulations. This is reasonable, since simulations showed no significant differences when $p_{0}$ was varied by $\pm 30 \mathrm{hPa}$ while the temperature $T_{C A}$ was kept constant.

\section{Treatment of vortex decay}

As stated earlier we prefer 2D simulations for the sake of saving computing time. Unfortunately, the most efficient vortex decay mechanism, the Crow instability (CROW, 1970), is a 3D phenomenon, hence cannot be simulated in 2D. In order to cure this deficiency of $2 \mathrm{D}$ simulations we have implemented a circulation control tool (CC-tool) to artificially enforce and monitor an accurate vortex decay. This method is based on results by HolzÄPFEL $(2003,2006)$ on vortex decay. The CC-tool tracks the vortex cores and computes the circulation, depending on the current circulation the diffusion is artificially increased in the neighborhood of the vortex securing a proper vortex decay. The microphysical properties of a contrail depend on how fast the vortex pair sinks $(w(t))$ and how long it lives ( $\left.t_{\text {breakup }}\right)$ as the ice crystals trapped in the vortex may face subsaturation due to adiabatic compression and eventually sublimate.

During the jet phase (roughly the first $20 \mathrm{~s}$ ) the circulation around the wings (Kutta-Joukowski law) is transformed into a pair of counterrotating vortices with downdraughts inside and updraughts outside of the corresponding vortex centres which are $b_{0} / 2$ off the fuse-
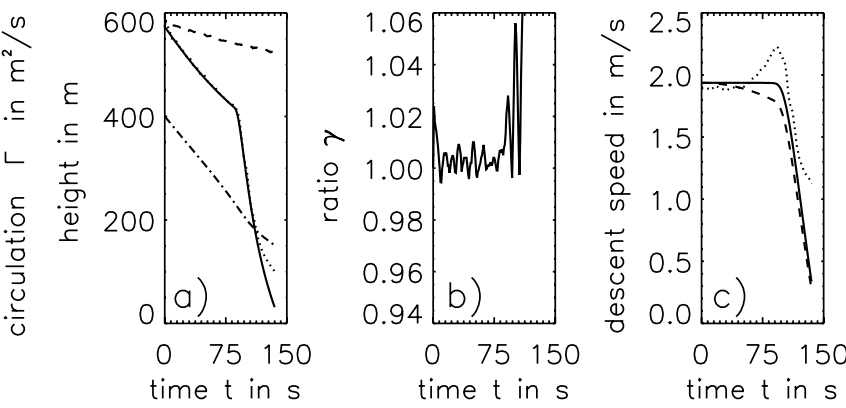

Figure 1: a) Evolution of the circulation $\Gamma_{5-15}$ for the baseline case $\left(\Gamma_{C C}\right.$, solid line; $\Gamma_{n u m}$, dotted line; $\Gamma_{\text {num }}$ with $\mathrm{CC}$-tool turned off, dashed line). The dash-dotted line displays the evolution of the height of the vortex center. b) Ratio $\gamma=\Gamma_{\text {num }} / \Gamma_{C C}$ vs. time is displayed. c) Descent speeds vs time are displayed. The solid/dashed line shows the theoretical evolution (excluding/including buoyancy). The dotted line shows the simulated values.

lage. The two vortices mutually induce an initial downward transport at a speed $w_{0}$, approximated by:

$$
v_{\theta}\left(b_{0}\right) \approx \frac{\Gamma_{0}}{2 \pi b_{0}}=: w_{0} .
$$

The relationship of circulation and descent speed is not linear, as (3.1) might suggest. The shape of the radial velocity profile changes, as the critical radius $r_{c}$ increases. Typically the circulation initially decreases without considerably slowing down the downward movement. Buoyancy effects may become important in a strongly stratified environment. The restoring force can become large at late stages (great vertical displacement of the vortex), such that the vortex motion halts or even reverses. Baroclinic instability around the upper stagnation point of the vortex system leads to detrainment of air that, being warmer than its environment, starts to rise. A buoyant plume arises in the vertical along the pathway of the sinking vortex pair.

For a single vortex its circulation is evaluated integrating the vorticity $\eta=\frac{\partial w}{\partial x}-\frac{\partial u}{\partial z}$ over the whole domain. For a two-vortex system, the integration domain must be restricted to the vicinity of the according vortex centre in order not to include opposite-sign contributions of the counterrotating vortex:

$$
\Gamma_{r}=\int_{0}^{r} \int_{0}^{2 \pi} \eta\left(r^{\prime}, \phi\right) r^{\prime} d \phi d r^{\prime} .
$$

In our analysis circulation is given in terms of a radius-averaged measure (using the discrete levels $r=\{5 \mathrm{~m}, . ., 15 \mathrm{~m}\}), \quad \Gamma_{5-15}=\frac{1}{11} \sum_{r=5 \mathrm{~m}}^{15 \mathrm{~m}} \Gamma_{r}$ (following HOLZÄPFEL, 2003). Starting a simulation with $\Gamma_{0}=$ $650 \mathrm{~m}^{2} / \mathrm{s}$ corresponds to a $\Gamma_{5-15}$-value of about $\Gamma_{0}=$ $575 \mathrm{~m}^{2} / \mathrm{s}$, since not all circulation is contained within the inner circles. The decay of the vortex pair depends on meteorological parameters (stratification $N_{B V}$ and eddy 

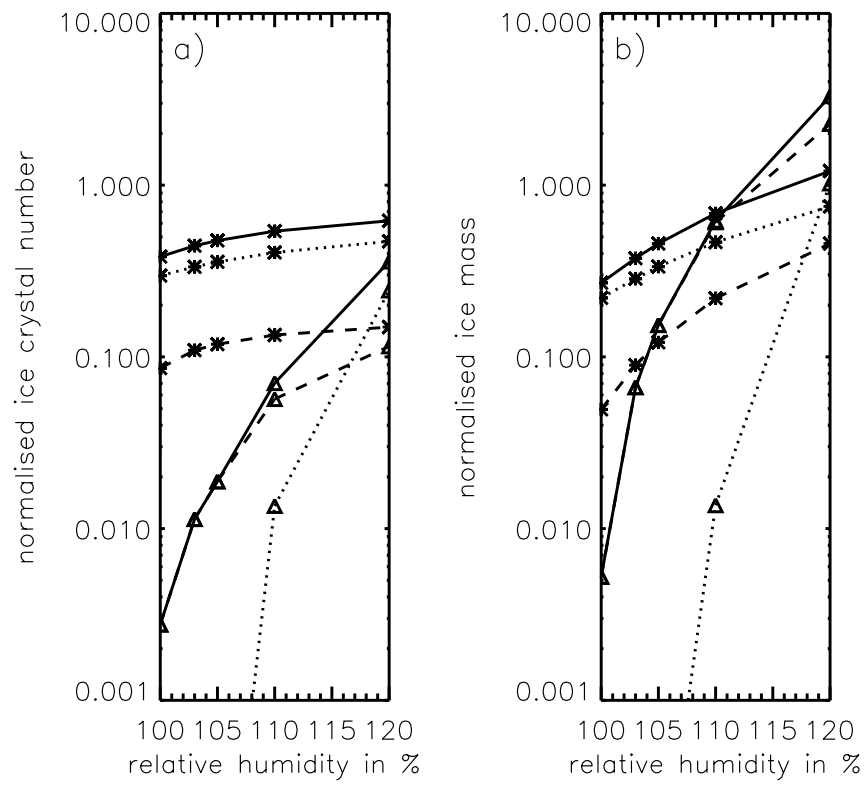

Figure 2: Normalised totals of surviving ice crystal number (left) and ice mass (right) as a function of relative humidity. The curves are shown for two temperatures $\left(T_{C A}=222 \mathrm{~K}\right.$, triangles; $T_{C A}=209 \mathrm{~K}$, asterisks). The linestyle denotes the integration over the total crosssection (solid), primary (dotted) and secondary (dashed) wake.

dissipation rate $\varepsilon$ ) as well as on aircraft parameters (initial circulation $\Gamma_{0}$ and initial vortex separation $b_{0}$ ). The vortex decay occurs in two phases. During the diffusion phase the vortex pair weakens slowly, independent of the meteorological parameters. After a certain onset time $T_{2}^{*}$ (using the definition of HoLZÄPFEL, 2006) a phase of rapid decay sets in. Generally, $T_{2}^{*}$ is smaller and the rapid decay is faster for stronger turbulence and for more stable stratification. A stronger circulation for the same aircraft geometry $b_{0}$ (e.g. higher payload) leads to a faster break-up, since the interaction of the two vortices is more intense. The temporal evolution of $\Gamma_{5-15}$ is parametrised in HoLZÄPFEL (2003), henceforth labelled as $\Gamma_{C C}$. The end of the vortex phase $t_{\text {breakup }}$ is reached when the vorticity fluctuations due to the swirling vortices reaches ambient turbulence levels. In the simulations this is characterized by $\Gamma_{C C}$ dropping below $1 \%$ of the initial value.

The CC-tool tracks the vortex cores (their height for the baseline case is depicted in Figure 1a and evaluates the radius-averaged circulation $\Gamma_{5-15}$ which is denoted by $\Gamma_{\text {num }}$. Depending on the ratio $\gamma=\frac{\Gamma_{\text {num }}}{\Gamma_{C C}}$ the diffusion coefficient in the simulations is artificially increased around the vortex cores and adapted each second in order to ensure $\gamma$ to be close to 1 and to maintain a realistic decay. Figure 1a shows the evolution of the circulation $\Gamma_{5-15}$ for the baseline case. The solid line shows $\Gamma_{C C}$, the dotted one $\Gamma_{\text {num }}$ and the dashed one the simulation with the circulation control turned off (i.e. no increased diffusion). Simulations without a circula- tion control are obviously not adequate for our purpose as the decay is much too slow. Figure $1 \mathrm{~b}$ shows the ratio $\gamma$ vs. time. During the diffusion phase the simulated circulation deviates less than $2 \%$ from $\Gamma_{C C}$ and at the beginning of the rapid phase one still has good agreement with Holzäpfel's results. Close to break-up time $t_{\text {breakup }}, \Gamma_{\text {num }}$ is not reduced fast enough, since the diffusion cannot be further increased due to the diffusionstability criterion. In Figure 1c) the vortex descent speed for the baseline case is shown. The solid/dashed curves show Holzäpfel's benchmark values (ex- and including buoyancy effects, see HolzÄPFEL, 2003). The dotted curve shows the simulated values. Initially the downward transport is slightly underestimated and towards the end it is overestimated. However, the descent speeds are in good agreement with HOLZÄPFEL (2003) and the final vertical displacement is within the uncertainty range of Holzäpfel's results. Due to the too strong downward transport in the final stages, the sublimation of ice mass is overestimated. As a simple compensation measure, $N$ and $I W C$ at vortex breakup are evaluated roughly $5 s$ before $t_{\text {breakup }}$.

\section{Results}

This section presents the results of the numerical simulations. First we stipulate some definitions. The total normalised ice mass (per meter of flight path) is

$$
t I W C(t)=\iint I W C(x, z, t) d x d z / I W C_{0} .
$$

The total normalised number of ice crystals $t N$ is defined analogously. In order to determine the quantities $(N, I W C)$ inside the primary wake, the integral is taken over $r=50 \mathrm{~m}$ circles around the vortex centres:

$$
p I W C(t)=\left(\iint_{r_{\text {left }}} \int_{r_{\text {right }}<50 \mathrm{~m}} I W C(x, z, t) d x d z\right) / I W C_{0} .
$$

$p N$ (number of ice crystals in the primary wake) is computed in an analogous way. As stated earlier, a bouyant plume of ice arises between the original flight altitude and the upper stagnation point of the vortex pair (also called secondary wake). The ice mass in the secondary wake, $s I W C$, can be deduced by subtracting the primary ice mass $p I W C$ from the total ice mass $t I W C$; a corresponding procedure gives the number of crystals in the secondary wake, $s N$.

The term "surviving" ice crystals/mass refers to the time of vortex breakup (taking into account the $5 \mathrm{~s}$ correction, see section 3). All the results discussed in this section refer to baseline case simulations with specified 
temperature $T_{C A}$ and relative humidity $R H_{i}=1+s_{i}$ (supersaturation $s_{i}$ ), unless it is mentioned explicitly. In the following subsections the sensitivity of the spatial and temporal ice distribution on various parameters like relative humidity, temperature, ambient turbulence, stratification and aircraft weight is discussed.

\subsection{Variation of relative humidity}

The left panel of Fig. 2 shows the normalised number of surviving ice crystals as a function of relative humidity for two flight levels $\left(T_{C A}=222 \mathrm{~K}\right.$ : triangles, $T_{C A}=209 \mathrm{~K}$ : asterisks). Since there is no nucleation of new ice crystals, the normalised value must be less than unity. Not surprisingly, the moister the environment, the more crystals survive. The sensitivity of the surviving fraction to relative humidity increases with temperature. At the lower temperature crystal sublimation is slower than at the higher one, so that at $T_{C A}=209 \mathrm{~K}$ only slightly more crystals are lost at ice saturation (40\% surviving) than at $20 \%$ supersaturation (60\% surviving). At $T_{C A}=222 \mathrm{~K}$ this sensitivity is much larger $(0.3 \%$ vs. $30 \%$ ). In the cold case the primary vortex contains the dominant fraction of the crystals at all supersaturations considered. However, in the warm case (triangles) the primary wake only dominates for $s_{i}$ approaching $20 \%$. At lower supersaturation the fraction in the secondary wake clearly dominates, and is even the only fraction surviving below $5 \%$ supersaturation. In that case detrainment of ice from the sinking primary vortex ceases before $t_{\text {breakup }}$ is reached, which has the effect that the contrail does not achieve its full vertical extent.

The right panel of Fig. 2 shows accordingly the normalised surviving ice mass, which can exceed unity because of crystal growth. As all curves are steeper than their counterparts in the left panel, one sees that the ice mass evolution during the vortex phase is more sensitive to ambient supersaturation than the evolution of crystal number because with increasing supersaturation not only the number but also the individual mass of the surviving crystals increases. During the vortex phase, the ice mass initially increases by deposition growth of the crystals until all the excess moisture from the ambient supersaturation in the vortex system is consumed. At small ambient supersaturation the majority of ice crystals in the primary vortices experience subsaturation, hence it turns out that the surviving crystals have smaller mean mass than the ice crystal population had initially. In a moister environment, at $t_{\text {breakup }}$ the crystals still benefit from the substantial initial growth, hence the mean size of the surviving crystals can be larger the mean size of the initial population. Especially the ice mass in the secondary wake grows since it has significant exposure to the ambient supersaturated air. The earlier an ice crystal is detrained, the larger it will be in the end. The growth rate of the crystals in the secondary wake
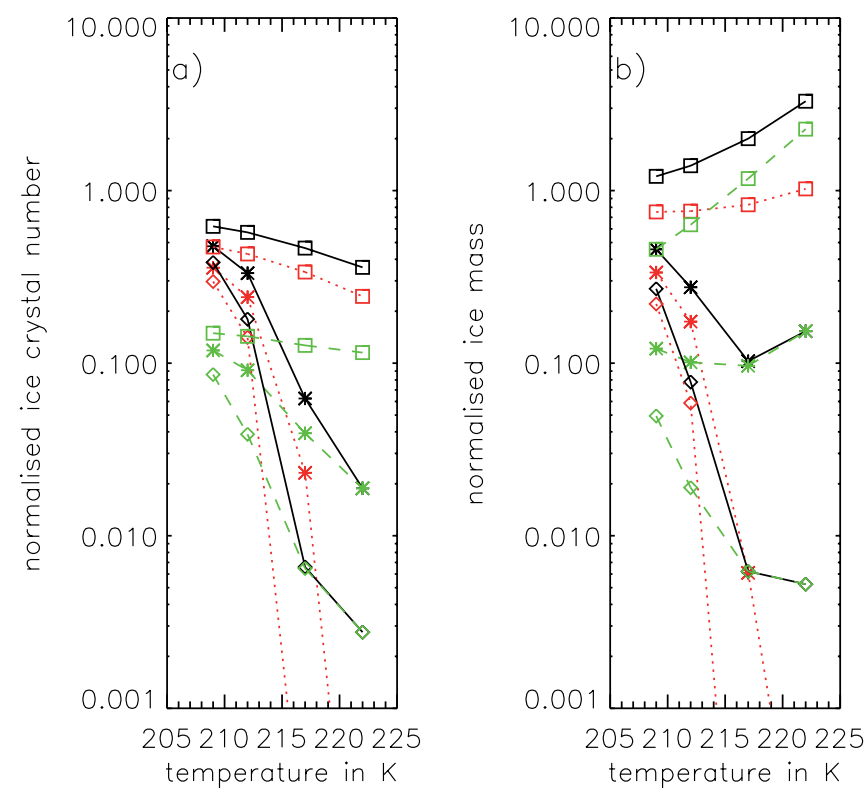

Figure 3: Normalised totals of surviving ice crystal number (left) and ice mass (right) as a function of temperature $T_{C A}$. The curves are shown for three relative humidities $\left(R H_{i}=120 \%\right.$, squares; $R H_{i}=$ $105 \%$, asterisks; $R H_{i}=100 \%$, diamonds). The colour and linestyle denote the integration over the total cross-section (black,solid), primary (red,dotted) and secondary (green,dashed) wake.

is larger in the warmer than in the colder case, since the absolute humidity is higher. In the warm case at all simulated ambient supersaturations the major part of ice mass is in the secondary wake, although at $s_{i}=20 \%$ the major fraction by number is in the primary wake. In the cold case the major fraction of ice is in the primary wake, both by number and by mass.

\subsection{Variation of temperature or flight altitude}

Fig. 3 reveals more clearly the influence of temperature or flight level, respectively, on ice mass and number concentration. Results are shown for three different supersaturations $\left(R H_{i}=120 \%\right.$ : squares, $R H_{i}=105 \%$ : asterisks, $R H_{i}=100 \%$ : diamonds). The total number of surviving ice crystals (black/solid lines, left) decreases with increasing temperature. While the temperature influence is weak in the moistest case $\left(s_{i}=20 \%\right)$ it is very strong in the slightly supersaturated cases, in particular in the primary wake, which totally vanishes when $T_{C A}$ is too high. In these cases the persistent contrail is made up exclusively of the ice in the secondary wake. However, also the number of crystals in the secondary wakes decreases with increasing temperature, because the source (i.e. the ice in the primary vortex) runs dry before the vortex reaches its final vertical position.

On the right panel the surviving ice mass is displayed. Comparing both graphs one sees that in the mass budget the increasing loss of ice crystals with increasing 
Table 1: Breakup time and fraction of surving ice crystals as a function of ambient turbulence.

\begin{tabular}{ccccc}
\hline$\varepsilon$ in $\mathrm{m}^{2} / \mathrm{s}^{3}$ & $3.5 \cdot 10^{-3}$ & $3.5 \cdot 10^{-4}$ & $3.5 \cdot 10^{-5}$ & $3.5 \cdot 10^{-6}$ \\
$t_{\text {breakup }}$ in s & 100 & 118 & 135 & 150 \\
$t N$ & 0.28 & 0.15 & 0.062 & 0.030 \\
\hline
\end{tabular}

Table 2: Various effects of varying stratification on vortex break-up and number of ice crystals.

\begin{tabular}{cccc}
\hline$N_{B V}$ in s & 0 & $10^{-2}$ & $2 \cdot 10^{-2}$ \\
$t_{\text {breakup }}$ in s & 165 & 135 & 117 \\
$\Delta z_{\text {breakup }}$ in $\mathrm{m}$ & 300 & 250 & 200 \\
$t N$ & 0.013 & 0.062 & 0.33 \\
$t N\left(s_{i}=10 \%\right)$ & 0.04 & 0.41 & 0.47 \\
$t N(T=209 \mathrm{~K})$ & 0.28 & 0.41 & 0.59 \\
\hline
\end{tabular}

temperatures is partly balanced by an increased growth rate of the surviving ice crystals, which is apparent in the curves that increase with increasing temperature. In particular the ice in the secondary wake contributes to this effect. This can lead to a higer total ice mass at the upper end of the considered temperature range, although the number of crystals strictly decreases with increasing $T_{C A}$.

\subsection{Variation of ambient turbulence}

In the following three subsections parameters affecting the breakup time $t_{\text {breakup }}$ are varied, namely the ambient turbulence, the stratification, and the initial circulation. First, the impact of ambient turbulence given in terms of eddy dissipation rate $\varepsilon$ is investigated for $T_{C A}=217 \mathrm{~K}$ and $s_{i}=5 \%$. Stronger ambient turbulence leads to faster decay of the vortex system (reduction of $t_{\text {breakup }}$; see Table 1) and smaller vertical displacement. The studied turbulence strengths cover the full range of null $\left(\varepsilon=3.5 \cdot 10^{-6} \mathrm{~m}^{2} / \mathrm{s}^{3}\right)$, medium-strength $(\varepsilon=$ $\left.3.5 \cdot 10^{-4} \mathrm{~m}^{2} / \mathrm{s}^{3}\right)$ and extreme $\left(\varepsilon=3.5 \cdot 10^{-3} \mathrm{~m}^{2} / \mathrm{s}^{3}\right)$ turbulence events. We employ the upperlevel turbulence classification (valid for heights above flight level 200) from SHARMAN (2005). Figures 4a, b show vertical profiles of horizontally integrated $N$ and $I W C$ for a variety of eddy dissipation rates. The black curves, included for better orientation, show the vertical profile of the initial distribution of $N$ and $I W C$, that is, crystals uniformly distributed over two circles (one per wing) with radius $r=20 \mathrm{~m}$ at height $z_{C A}=400 \mathrm{~m}$. With decreasing turbulence strength the fraction $t N$ of surviving ice crystals gets smaller (see Table 1), in particular in the primary wake, because the vortices exist longer and sink down farther. The variation of the vortex sinking with different turbulence levels also affects the number and mass of ice in the secondary wake, as can be seen in the figure.

\subsection{Variation of stratification}

The subsequent set of simulations shows the impact of stratification, again computed with $T_{C A}=217 \mathrm{~K}$ and $s_{i}=5 \%$. $\varepsilon$ is kept constant at $3.5 \cdot 10^{-5} \mathrm{~m}^{2} / \mathrm{s}^{3}$. Runs with three different stratifications (neutral, baseline case and strongly stable atmosphere) are compared (for values of $N_{B V}$ see Table 2). For $N_{B V}=0$, the standard domain was enlarged in the vertical direction, in order to avoid boundary effects of the far-travelling vortex pair.

According to HOLZÄPFEL (2003) the vortex pair decays faster in a more stable atmosphere, hence more ice should survive. With increasing stability the vertical extent of the contrail is decreased, since the vortex breaks up earlier and the buoyancy drag additionally retards the descent. Figures $4 c$, d show the vertical profiles of horizontally integrated $N$ and $I W C$, with the initial distributions (black lines) included for orientation as before.

Let us consider first some features of the strongly stable case (blue curves). The reduced downward displacement of the primary vortex is apparent (see Table 2). The detrained air parcels are highly buoyant (updraughts up to $w=4 \mathrm{~m} / \mathrm{s}$ ), such that the secondary wake even reaches altitudes above the initial flight level. The strength of the baroclinic instability at the upper stagnation point increases with increasing stability, such that the number of ice crystals in the secondary wake becomes as high as in the primary wake.

In case of a neutral atmosphere, the ice in the primary wake is completely lost. The vortex cores have moved down to $z=100 \mathrm{~m}$ and the small bump in ice concentration around $z=150 \mathrm{~m}$ is formed of detrained particles, that is, it does not belong to the the primary wake. The ice concentration in the secondary wake decreases with downward distance from flight level in a roughly exponential fashion.

The total number of surviving crystals varies between $1.3 \%$ (neutral) and $33 \%$ (strong stability), which shows that the stratification of the ambient atmosphere is an important parameter for contrail evolution. It must be, however, remarked, that this sensitivity is weaker when it is colder or moister than in the cases considered above (see $t N\left(T_{C A}=209 \mathrm{~K}\right)$ and $t N\left(s_{i}=10 \%\right)$ in Table 2).

, blue; $\Gamma_{0}=650 \mathrm{~m}^{2} / \mathrm{s}$, red; $\Gamma_{0}=700 \mathrm{~m}^{2} / \mathrm{s}$, green).

\subsection{Variation of initial circulation}

The initial circulation of each vortex, $\Gamma_{0}$, is proportional to the weight of the aircraft which changes during flight due to fuel consumption (note that the fuel weight contributes a significant part to the overall weight of an aircraft). Here, we test how a variation of $\Gamma_{0}$ that is typical for the range of fuel filling levels during a flight affects the contrail's vertical profiles of ice mass and number concentration. Ambient conditions are as in the previous subsection. Aircraft geometry and speed are unchanged. 

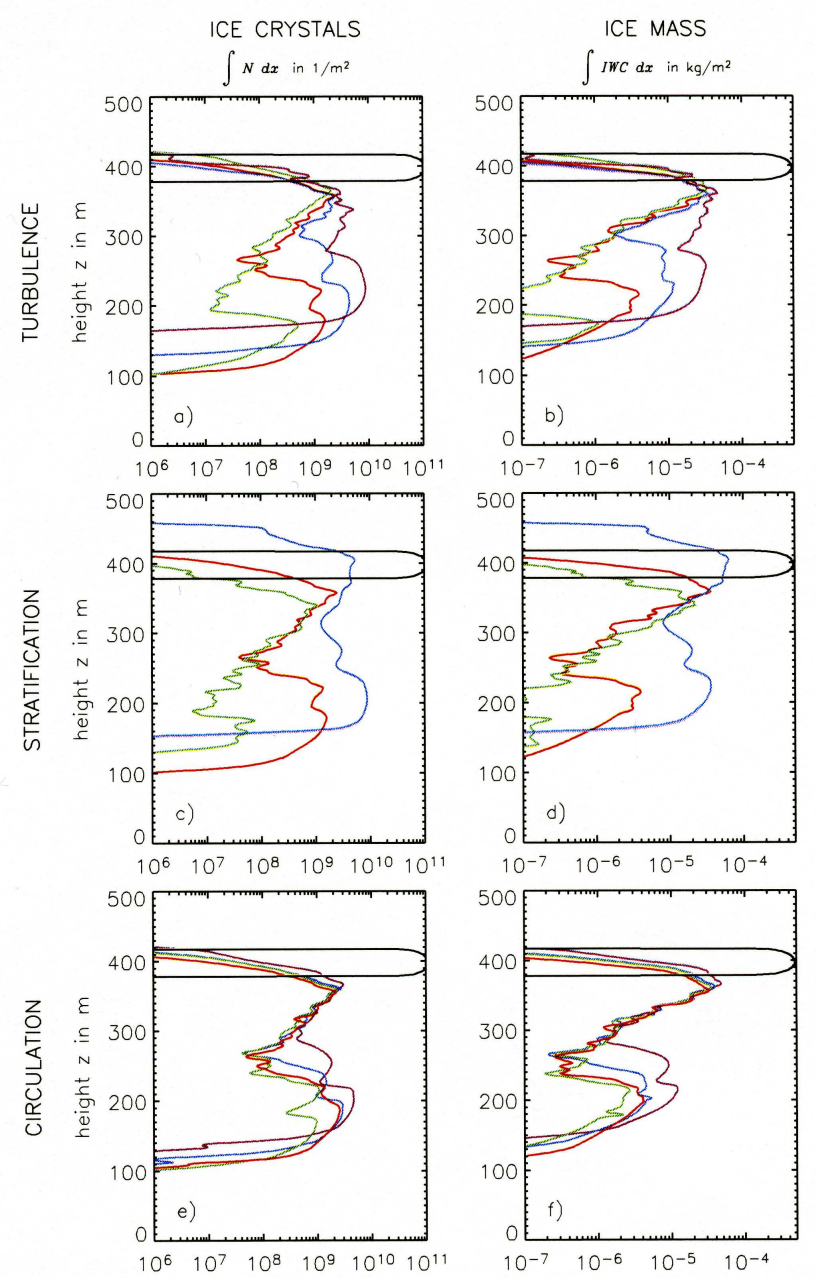

Figure 4: Vertical ice crystal number (left) and mass (right) profile for various simulations at vortex breakup time. The black lines show the initial distribution of ice crystal number and mass at $z_{C A}=400 \mathrm{~m}$. a) and b) effect of turbulence level $\left(\varepsilon=3.5 \cdot 10^{-6} \mathrm{~m}^{2} / \mathrm{s}^{3}\right.$, green; $\varepsilon=3.5 \cdot 10^{-5} \mathrm{~m}^{2} / \mathrm{s}^{3}$, red; $\varepsilon=3.5 \cdot 10^{-4} \mathrm{~m}^{2} / \mathrm{s}^{3}$, blue; $\varepsilon=3.5$. $10^{-3} \mathrm{~m}^{2} / \mathrm{s}^{3}$, purple). c) and d) effect of stratification $\left(N_{B V}=0 \mathrm{~s}^{-1}\right.$, green; $N_{B V}=10^{-2} s^{-1}$, red; $N_{B V}=2 \cdot 10^{-2} s^{-1}$, blue). e) and f) effect of circulation $\left(\Gamma_{0}=500 \mathrm{~m}^{2} / \mathrm{s}\right.$, purple; $\Gamma_{0}=600 \mathrm{~m}^{2} / \mathrm{s}$

The results are given in Table 3 and the profiles are presented in Figures $4 \mathrm{e}, \mathrm{f}$ ).

During flight, when $\Gamma_{0}$ decreases, the life time of the vortex pair increases, since the interaction of the two vortices gets weaker with decreasing $\Gamma_{0}$. This has two main effects, (1) later onset of the rapid decay phase and (2) slower descent. The dominant impact of descent speed on the final vertical displacement $\Delta z_{\text {breakup }}$ (see Table 3 ) is partially balanced by the changed vortex life time.

The vertical profiles of $N$ and IWC (horizontally integrated, see Fig. 4e, f) differ only weakly for the inflight variation of the circulation. In the present case, the total number of surviving crystals $t N$ varies from $5.6 \%$ for the largest circulation (fuel filling level high) to $13 \%$ for the smallest circulation (fuel filling level low). Thus, the effect of the initial circulation on contrail evolution is much smaller than the effect of the other factors discussed before.

Table 3: Various effects of varying initial circulation $\Gamma_{0}$ on vortex break-up and number of ice crystals.

\begin{tabular}{ccccc}
\hline$\Gamma{\text { in } \mathrm{m}^{2} / \mathrm{s}}$ & 500 & 600 & 650 & 700 \\
$t_{\text {breakup }}$ in s & 160 & 140 & 135 & 128 \\
$\Delta z_{\text {breakup }}$ in $\mathrm{m}$ & 215 & 230 & 250 & 260 \\
$t N$ & 0.13 & 0.089 & 0.062 & 0.056 \\
\hline
\end{tabular}

\section{Discussion}

\subsection{Impact of the temperature and relative humidity variation - theoretical background}

The results of the preceeding section revealed that the number of surving ice crystals depends critically on ambient humidity as well as on ambient temperature. Here we want to give some theoretical explanation for the simulation results.

During the downward transport the air trapped inside the vortex undergoes adiabatic heating. We can estimate the vertical displacement $\delta z_{\text {crit }}$ where the relative humidity falls below saturation, when for now we simply assume that the ice crystals do not grow or evaporate during the vortex falling. Then the air within the vortex heats up according to a dry adiabatic process, such that $\delta T=\Gamma_{d} \delta z$ with $\Gamma_{d}=g / c_{p} \approx 9.8 \mathrm{~K} / \mathrm{km}$ independently of the ambient lapse rate. It does not make sense to use an ice adiabatic lapse rate, because the ice is not in equilibrium with the vapour, and the ice mass sublimating is so small that the release of latent heat only weakly affects the lapse rate. Also, the effect of the stratification on the pressure profile and thus on the lapse rate is negligible. The total amount of vapour of an initially supersaturated air parcel at cruise altitude matches the one of a saturated parcel at some higher temperature, namely:

$$
\left(1+s_{i}\right) q_{s}\left(T_{C A}\right)=q_{s}\left(T_{C A}+\Gamma_{d} \delta z_{c r i t}\right) .
$$

Here, $q_{s}$ is the saturation mixing ratio of water vapour (with respect to ice). Taylor expansion of the rhs and using $\frac{\partial}{\partial T} \ln q_{s}=0.9 \frac{\partial}{\partial T} \ln e_{s}$ (the constant 0.9 accounts for the adiabatic atmospheric pressure change during the descent of the plume, and $e_{s}$ is saturation vapour pressure) gives:

$$
\delta z_{c r i t}=\frac{s_{i}}{\Gamma_{d} \cdot 0.9 \frac{\partial}{\partial T}\left(\ln e_{s}\left(T_{C A}\right)\right)} .
$$

The threshold is $\delta z_{c r i t}=s_{i} \cdot 750 \mathrm{~m}$ for cold conditions $\left(T_{C A}=205 \mathrm{~K}\right)$ and $s_{i} \cdot 910 \mathrm{~m}$ for warm conditions $\left(T_{C A}=\right.$ 


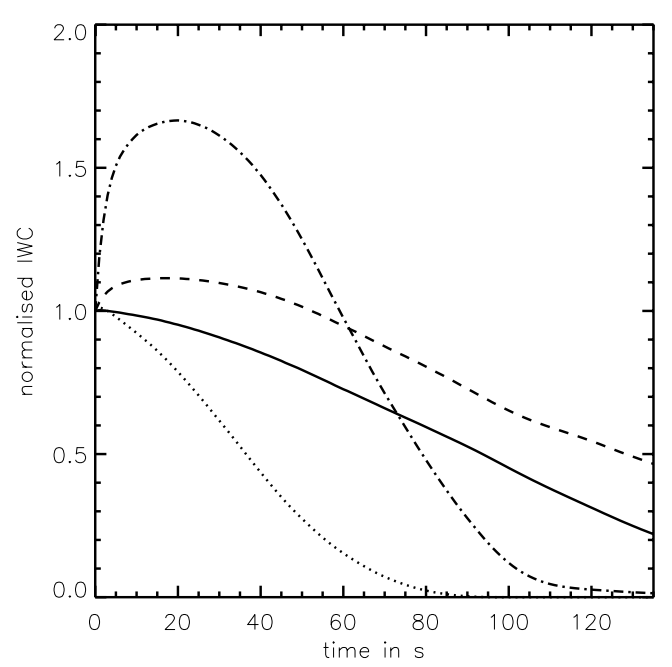

Figure 5: Temporal evolution of primary ice mass for various temperatures and relative humidities $\left(T_{C A}=222 \mathrm{~K}\right.$ and $s_{i}=10 \%$, dashdotted; $T_{C A}=209 \mathrm{~K}$ and $s_{i}=10 \%$, dashed; $T_{C A}=209 \mathrm{~K}$ and $s_{i}=0 \%$, solid; $T_{C A}=222 \mathrm{~K}$ and $s_{i}=0 \%$, dotted).
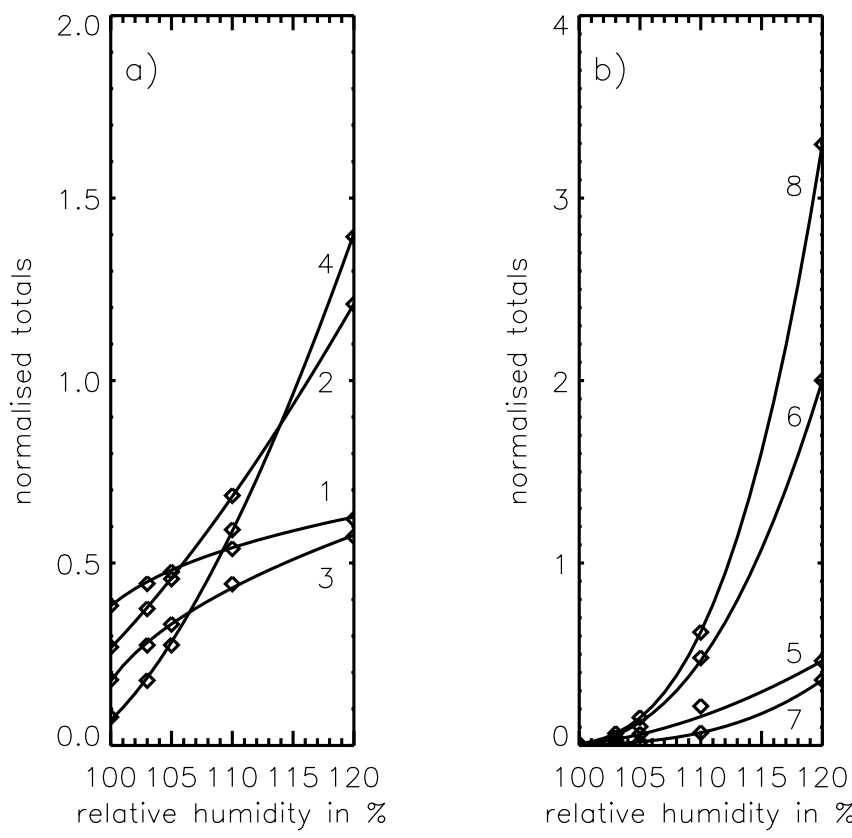

Figure 6: Power law fits (see equation 5.1) for the surviving number and mass of ice crystals vs. relative humidity for different temperatures. The curve labels correspond to the line number in Table 4. Diamonds represent the results from the simulations.

$225 \mathrm{~K})$. Threshold values numerically computed with the full nonlinear equations are about $s_{i} \cdot 50 \mathrm{~m}$ smaller. For our moistest case this means that subsaturation is reached in the vortex at a vertical distance of less than about $180 \mathrm{~m}$, a distance that is reached in all our simulations. Hence, all our simulations reach subsaturation. The total ice mass does not fall below its initial value as long as the plume is displaced less than $\delta z_{c r i t}$. Actually the ice crystals grow during the first 10 to $40 \mathrm{~s}$ until all excess vapour from the ambient supersaturation in the plume is condensed. The rate of deposition increases considerably with temperature. Initially, the total ice mass per flight path is $1.46 \cdot 10^{-2} \mathrm{~kg} / \mathrm{m}$ (water emitted by the engines). The excess ambient moisture inside the plume with radius $r_{p}=20 \mathrm{~m}$ is $\delta q=s_{i} q_{s}(T) 2 r_{p}^{2} \pi \rho_{\text {air }}$. For $s_{i}=10 \%$ and $T_{C A}=220 \mathrm{~K}, \delta q=0.66 \mathrm{~kg} / \mathrm{m}$. Varying $T_{C A}$ within the simulated range can in/decrease $\delta q$ by a factor of 2-3. Thus, the total ice mass can increase by $20 \%$ (cold, $5 \%$ supersaturation) up to $300 \%$ (warm, $20 \%$ supersaturation). Since there is no nucleation during the vortex phase, the initially existing crystals can grow substantially.

The sinking vortex pair reaches $\delta z_{c r i t}$ some time after all the excess vapour is condensed. The plume air is subsaturated and the crystals lose mass in order to maintain saturation. Typically, the relative humidity is about 95-98\% inside the plume, as the sublimation lags behind the adiabatic heating. The sublimation is faster for higher temperatures, but more ice is necessary to saturate the air. How far below the threshold altitude $z_{C A}-\delta z_{c r i t}$ the ice crystals survive, depends most sensitively on temperature and cannot be easily answered by theoretical reasoning. Hence we resort to the presented numerical simulations.

In Fig. 5 the temperature sensitivity of the sublimation process is demonstrated. The temporal evolution of the primary ice mass is shown for $T_{C A}=\{209,222\} \mathrm{K}$ and $s_{i}=0 \%, 10 \%$. For $s_{i}=0 \%$, the displacement threshold $\delta z_{\text {crit }}$ is $0 \mathrm{~m}$ and subsaturation sets in immediately. For $T_{C A}=222 \mathrm{~K}$ the ice has been completely sublimated within $100 \mathrm{~s}$, whereas for $T_{C A}=209 \mathrm{~K}$ still more than $20 \%$ of the ice mass surives the vortex phase $\left(t_{\text {breakup }}=135 \mathrm{~s}\right)$. At $s_{i}=10 \%$, it takes about $t_{\text {crit }}=40 \mathrm{~s}$ until the vortex falls below the critical height $\left(\delta z_{\text {crit }}=\right.$ $75 \mathrm{~m}$ ), hence it takes at least $40 \mathrm{~s}$ more than in the other cases $\left(s_{i}=0 \%\right)$ to sublimate the ice completely. At $T_{C A}=222 \mathrm{~K}$ the estimated minimum survival time of the crystals $(100 \mathrm{~s}+40 \mathrm{~s})$ is close to $t_{\text {breakup }}$. It turns out that about $1 \%$ of ice mass and crystals (not shown here) survive. At $T_{C A}=209 \mathrm{~K}$, the initial condensation of excess moisture is slower than at $T_{C A}=222 \mathrm{~K}$, nevertheless the colder plume contains more ice mass at $t>70 \mathrm{~s}$ and nearly $50 \%$ of the ice mass still exist at vortex break-up. We can conclude that the survival of the ice in the primary vortex depends on the interrelationship of different timescales, namely $t_{\text {crit }}$ (most sensitive to $s_{i}$ ), $t_{\text {breakup }}$ (depending on aircraft parameters, stratification and ambient turbulence), and the growth/sublimation timescale of the ice crystal population (most sensitive to $T$ ).

$\delta z_{c r i t}$ was eventually reached in all our simulations before vortex breakup. The question then arises whether there are cases where this does not occur, and where therefore all ice crystals should survive. This could happen for instance in highly supersaturated air, but then natural cirrus may have formed anyway. Another pos- 
Table 4: Fit parameters for equation 5.1 at various temperatures.

\begin{tabular}{ccccc}
\hline No. & quantity & $y_{0}$ & $\beta$ & $\alpha$ \\
\hline 1 & $t N$ at $T=209 \mathrm{~K}$ & $3.82 \cdot 10^{-1}$ & $96.5 \%$ & 0.25 \\
2 & $t I W C$ at $T=209 \mathrm{~K}$ & $2.67 \cdot 10^{-1}$ & $89.3 \%$ & 1.43 \\
3 & $t N$ at $T=212 \mathrm{~K}$ & $1.76 \cdot 10^{-1}$ & $98.3 \%$ & 0.47 \\
4 & $t I W C$ at $T=212 \mathrm{~K}$ & $0.69 \cdot 10^{-1}$ & $96.5 \%$ & 1.56 \\
5 & $t N$ at $T=217 \mathrm{~K}$ & $6.59 \cdot 10^{-3}$ & $98.0 \%$ & 1.77 \\
6 & $t I W C$ at $T=217 \mathrm{~K}$ & $2.79 \cdot 10^{-3}$ & $99.0 \%$ & 2.14 \\
7 & $t N$ at $T=222 \mathrm{~K}$ & $2.69 \cdot 10^{-3}$ & $93.8 \%$ & 3.4 \\
8 & $t I W C$ at $T=222 \mathrm{~K}$ & $9.18 \cdot 10^{-3}$ & $96.9 \%$ & 2.95 \\
\hline
\end{tabular}

Table 5: Ice crystal number density in a plume of $3 \mathrm{~s}$ age as a function of ambient temperature (taken from Fig. 4 in KÄRCHER et al., 1998).

\begin{tabular}{ccccc}
\hline$T_{C A}$ in $K$ & 209 & 212 & 217 & 222 \\
$N$ in $10^{4} \mathrm{~cm}^{-3}$ & 15 & 12 & 8 & 1 \\
\hline
\end{tabular}

sibility is given for smaller aircraft than the simulated one, when the vortices sink slower than here such that their maximum downward travelling distance is less than $\delta z_{\text {crit }}$.

\subsection{Power-law fitting}

The number and mass concentrations of ice crystals surviving the vortex phase increases monotonically with ambient relative humidity. It turns out that the dependence can be fitted with a power law. The function $f\left(R H_{i}\right)$ approximates the normalised quantities $t I W C$ and $t N$ for the baseline scenario:

$$
f\left(R H_{i}\right)=y_{0}\left(\frac{R H_{i}-\beta}{100 \%-\beta}\right)^{\alpha} .
$$

The three parameters $y_{0}, \beta, \alpha$ are fitted (chi-square) for $t I W C$ and $t N$ using the simulation results at the five humidity levels $R H_{i}=100 \%, 103 \%, 105 \%, 110 \%$, $120 \%$. The parameter values for all temperatures $T_{C A}$ are listed in Table 5. At most data points the absolute error $\left|f\left(R H_{i}\right)-t N\left(R H_{i}\right)\right|$ and $\left|f\left(R H_{i}\right)-t I W C\left(R H_{i}\right)\right|$ is below 0.005 . The simulation results and the corresponding approximations are shown in Fig. 6.

In equation 5.1, $y_{0}$ is the surviving fraction (mass or number) when the environment is just saturated. As seen from the table, $y_{0}$ decreases strongly with increasing temperature. The parameter $\beta$ is that relative humidity where $f$ becomes zero, i.e., the ice sublimates completely during the vortex phase. As the table shows, all values of $\beta$ range from about 90 to $99 \%$, consistent with the fact that a contrail does not survive significantly after the end of the vortex phase even when the air is only slightly subsaturated. The exponents $\alpha$ increase strongly with increasing temperature which expresses the fact that the sensitivity of $t I W C$ and $t N$ on ambient humidity increases strongly with increasing temperature.

\subsection{Testing minor sensitivity parameters}

In our simulations we have used a fixed number of ice crystals per meter of flight, in order to allow the same normalisation for all simulations. Actually, this number can vary within about one order of magnitude, depending for instance on ambient temperature, fuel sulphur content, soot particle emission index for various engines etc. For testing the sensitivity of the results with regard to the ice crystal number density we have taken from KÄRCHER et al. (1998) temperature-dependent estimates of $N$ assuming a medium sulphur content. These are listed in Table 5. The temperature-dependent ice number density equals our standard choice (8. $10^{4} \mathrm{~cm}^{-3}$ ) at $T_{C A}=217 \mathrm{~K} . N$ is higher at colder and considerably smaller at higher temperature. Simulations with the varied $N$ and $s_{i}=5 \%$ were performed for the two cold cases, yet it was found that the normalised number of surviving ice crystals and mass do not change significantly compared to our standard choice of $N$. However at $T_{C A}=222 \mathrm{~K}$ there is a non-negligible sensitivity to $N$ : a larger fraction of crystals survive when less crystals are present initially.

Fig. 7 shows the fraction of surviving crystals and ice mass for the warm case $\left(T_{C A}=222 \mathrm{~K}\right)$ at supersaturations ranging from 0 to $20 \%$, comparing the runs with our standard $N$ and the one corresponding to $T_{C A}=$ $222 \mathrm{~K}$ from Table 5. Note the large factor of eight between these two ice number concentrations. We see that with $N=1 \cdot 10^{4} \mathrm{~cm}^{-3}$ considerably more crystals survive both, in the primary and secondary wake, than in the standard cases. The threshold supersaturation at which, say, $1 \%$ of ice crystals survives in the vortices is higher when the initial ice number density is lower. Nevertheless, the absolute number of surviving ice crystals is higher for the standard $N$ than for the smaller value of $N$ (due to the large difference between these two values). More excess vapour from the ambient supersaturation is deposited on the detrained crystals in the case with standard $N$ than in the other case. Thus, the secondary wake is stronger and the total surviving ice mass fraction is larger in the standard cases than in the cases with the small value of $N$.

We investigated several more modifications of the initial ice distribution. We introduced random density fluctuations of up to $\pm 30 \%$ on the uniform distribution. This had no impact and the concentration is smoothed out quickly, since the diffusion is very high (controlled by the CC-tool). Generally, we found out that the choice of distribution is not critical as long as the fraction of de/entrained particles is similar.

A vortex features a low pressure at the centre to compensate for centrifugal forces. LEWELLEN and 
LEWELLEN (2001) report of a typical underpressure of about $p_{v}=2 \mathrm{hPa}$. Accordingly, the temperature in a vortex core must be lower than at its limb; if we assume an adiabat, a cooling by $T_{v} \approx 0.5 \mathrm{~K}$ would result. Cooling, in turn, reduces the saturation vapour pressure and the sublimation rate during the descent of the vortex. A test simulation showed that the effect is negligible since the depression is confined to a small area of the primary wake.

\subsection{Comparison with recent 3D results}

Although 2D simulations have the advantage that one can test quickly a number of major and minor sensitivities, they have also some disadvantages compared with a full 3D model. The most obvious one is that the vortex decay process due to Crow instability can be achieved using numerical tricks, but the variations due to this instability along the flight path cannot be represented. However, the question is, is this variation so important that it is worth the effort of a full 3D model to compute it. To our view it is not. As stated before, the ultimate goal of this investigation is to provide initial conditions for later studies of the contrail-to-cirrus transition and the resulting contrail cirrus. For such a purpose the initial variations along the flight path, for example the mammatus like structures that often appear in supersaturated cases (an example can be found in GERZ et al. (1998), Fig. 2) are irrelevant since they will be forgotten hours later.

If we had a 3D simulation we would eventually average the results anyway. In this sense our 2D simulation is hoped to give results that are close to these desired averages. It is then possible that due to non-linearities this $2 \mathrm{D}$ average would be wrong (i.e. strongly differing from the true $3 \mathrm{D}$ average). A comparison with recent 3D results shows that this is not the case. We use the simulations of LEWELLEN and LEWELLEN (2001) and HUEBSCH and LEWELLEN (2008) for comparison. First, we note by looking at their drift-plots that there is not much along-flight-path variation in the cross-stream integrated fields during the vortex phase. Stronger variations evolve later in the beginning dispersion phase into which our simulations do not reach. As already said these variations will be smoothed away or otherway randomized by the atmospheric turbulence and shear motions and by crystal sedimentation in the course ot the transition to contrail cirrus.

LEWELLEN and LEWELLEN (2001) investigate cases of $2 \%$ up to $30 \%$ ice supersaturation, with $T=220 \mathrm{~K}$ (similar to ours), a slightly smaller lapse rate and turbulence than ours, but a larger crystal number density. A look at their Fig. 7 shows their vortex breakup times, which are similar to ours, and the surviving fraction of ice number. Let us consider the case
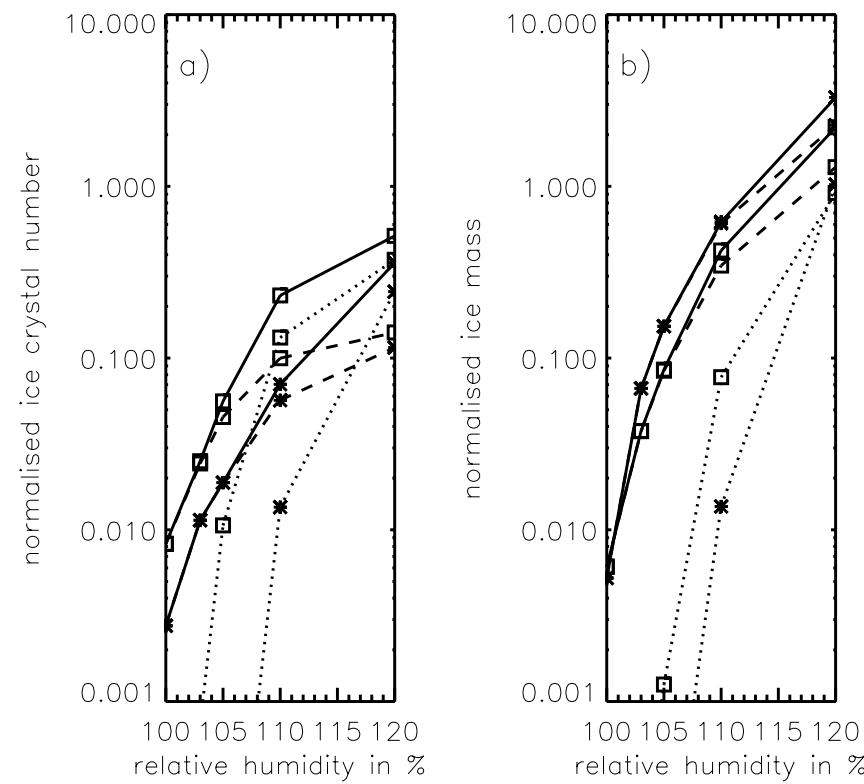

Figure 7: Normalised totals of surviving ice crystal number (left) and ice mass (right) as a function of relative humidity. The curves are shown for temperature $T_{C A}=222 \mathrm{~K}$ with initial ice crystal concentration $N=1 \cdot 10^{4} \mathrm{~cm}^{-3}$ (squares) and $N=8 \cdot 10^{4} \mathrm{~cm}^{-3}$ (asterisks). The line style denotes the integration over the total crosssection (solid), primary (dotted) and secondary (dashed) wake.

with $10 \%$ supersaturation. Our simulations give fractions $(t N)$ of 0.07 and 0.35 for 222 and $217 \mathrm{~K}$, respectively, whereas the 3D simulation at $220 \mathrm{~K}$ gives a fraction of 0.28 . At $20 \%$ supersaturation we got $t N$ of 0.21 and 0.46 for the two considered temperatures, whereas the 3D simulation gave $t N$ of 0.81 . Hence the differences are larger than for the less supersaturated case. However, this difference is most likely due to the differing assumptions in the microphysics parameterisations. HUEBSCH and LEWELLEN (2008) have shown that use of a monomodal size distribution as in LEWELLEN and LEWELLEN (2001) leads to overestimation of $t N$, so the value 0.81 is too large. Since we use a broad lognormal size distribution, our values of $t N$ are accordingly smaller than that of LEWELLEN and LEWELLEN (2001).

Summarising, in view of the later evolution into a contrail cirrus and the then dominant atmospheric motions the $3 \mathrm{D}$ variations in the early structure of a contrail are of minor importance. The surviving fraction of ice number compares well with those of recent 3D simulations when the conditions are similar. The ice mass is anyway determined by the ambient humidity. We conclude that a our 2D approach is accurate enough to compute the surviving fractions of ice number and mass and hence does justice to our next goal, to study contrail-tocirrus transformation. 


\section{Conclusions}

In the present paper we have used numerical 2D simulations of the contrail's vortex phase to determine major and minor factors of influence on the mass and number concentration of ice crystals that survive the vortex phase. As we had to perform a large number of simulations we decided not to use a 3D model but to instead equip a 2D model with a circulation control tool to ensure a realistic vortex decay. Surviving fractions and the final vertical profile of ice concentration are important initial conditions for studies of contrail-to-cirrus transformation. The simulations have been performed for only one aircraft type; the influence of wing span and flight speed has not yet been tested.

We can state the following conclusions:

- The fraction of ice number and mass that survives the vortex phase depends strongly on the ambient supersaturation with respect to ice. The dependence can be fitted by a power law. The dependence is strongest for the highest temperature that allows contrail formation (SchmidtAppleman criterion) and becomes weaker with decreasing temperature.

- Only the ice in the secondary vortex survives at low supersaturation, consistent with the results of SUSSMANN and GIERENS (1999), giving rise to a persistent yet very faint contrail.

- The stratification of the atmosphere and its turbulence level have strong impact of the fraction of the surviving ice via their dynamical effect on the sinking vortex pair. Strong turbulence leads to fast vortex decay, whereas weak turbulence allows the vortex pair to travel a long distance downward. Hence, in situations of strong turbulence more ice is rendered to the atmosphere than in weakly turbulent conditions. The downward travelling distance of the vortex increases with decreasing stratification strength, hence more crystals survive in more stable situations and vice versa. Additionally, more ice is detrained into the secondary wake in more stable situations.

- The initial number of ice crystals increases with decreasing ambient temperature within a range of about an order of magnitude. At the warmest temperatures that the Schmidt-Appleman criterion allows for contrail formation the surviving fraction is larger when less ice crystals are formed initially. However, the total number of surviving crystals and the surviving ice mass can be larger when more crystals are formed initially.

- The variation of the initial circulation with varying aircraft weight during a flight, details of the spatial distribution and the temperature profile within the vortices have only a minor influence on the surviving ice fraction.

We see that the structure and microphysical properties (hence also the optical appearance) of a contrail depend sensitively on ambient conditions during its early history up to the end of the vortex phase, which is about 2 to 3 minutes after exhaust. The most important conditions are atmospheric parameters, supersaturation, temperature, stability and turbulence level. We expect that also wind shear is an important parameter, but this has not yet been tested because it will affect the vortex evolution much stronger than the parameters studied so far, hence it deserves a separate study. With the information gained during the presented studies we are now able to reasonably initialise larger scale simulations of the contrail-to-cirrus transformation.

\section{Acknowledgments}

The authors would like to thank Bernd KärCHER, Thomas GERZ, and Frank HOLZÄPFEL for their help. The first author especially wishes to thank Andreas DÖRNBRACK for his steady support and many fruitful discussions. This work contributes to the DLR/HGFproject "Particles and Cirrus Clouds" (PAZI-2) and to the European Network of Excellence ECATS (Environmentally Compatible Air Transport System). The simulations have been carried out as a part of the special project "Ice-supersaturation and cirrus clouds" at ECMWF.

\section{References}

CROw, S. C., 1970: Stability theory for a pair of trailing vortices. - AIAA J. 8, 2172-2179.

GERZ, T., T. DÜRBECK, P. KONOPKA, 1998: Transport and effective diffusion of aircraft emissions. - J. Geophys. Res. 103, 25,905-25,913.

GERZ, T., T. EhrET, 1997: Wingtip Vortices and Exhaust Jets During the Jet Regime of Aircraft Wakes. - Aerosp. Sci. Technol. 7, 463-474.

GiERENS, K. M., 1996: Numerical simulations of persistent contrails. - J. Atmos. Sci. 53, 3333-3348.

Gierens, K., E. JENSEN, 1998: A numerical study of the contrail-to-cirrus transition. - Geophys. Res. Lett. 55, 4341-4344.

Heymsfield, A. J., J. Iaquinta, 2000: Cirrus crystal terminal velocities. - J. Atmos. Sci. 57, 916-938.

HolzäPfEL, F., 2003: Probabilistic two-phase wake vortex decay and transport model. - J. Aircraft 40, 323-331.

-, 2006: Probabilistic Two-Phase Aircraft Wake-Vortex Model: Further Development and Assessment. - J. Aircraft 43, 700-708.

HUEBSCH, W.W., D.C. Lewellen, 2008: Application of LES and binned microphysics for sensitivity study on contrail evolution. - Proc. of the TAC-Conference, June 26 to 29, 2006, Oxford, UK, 167-172. 
Intergovernmental PANel for Climate Change (IPCC), 1999: Aviation and the global atmosphere. J.E. PenNer et al. (Eds.). - Cambrige University Press, 373 pp.

Jensen, E., A.S. Ackerman, D.E. Stevens, O.B. Toon, P. Minnis, 1998: Spreading and growth of contrails in a sheared environment. - J. Geophys. Res. 103, 31557-31567.

KESSLER, E., 1969: On the distribution and continuity of water substance in atmospheric circulation. - Met. Mon. Amer. Meteor. Soc. 32, 80 pp. cher98

Kärcher, B., R. Busen, A. Petzold, F. P. Schröder, U. SCHUMANN, 1998: Physicochemistry of aircraftgenerated liquid aerosols, soot, and iceparticles: 2. Comparison with observations and sensitivity studies. - J. Geophys. Res. 103, 17129-17147.

KoENIG, L. R., 1971: Numerical modeling of ice deposition. - J. Atmos. Sci. 28, 226-237.

LEWELLEN, D. C., W. S. LEWELLEN, 2001: The effects of aircraft wake dynamics on contrail development. - J. Atmos. Sci. 58, 390-406.

MANNSTEIN, H., U. SCHUMANN, 2005: Aircraft induced contrail cirrus over Europe. - Meteorol. Z. 14, 549-554.

Mannstein, H., R. Meyer, P. Wendling, 1999: Operational detection of contrails from NOAA-AVHRR data. Int. J. Remote Sens. 20, 1641-1660.

SAUSEN, R., I. ISAKS $\overline{E N}$, V. GREWE, D. Hauglustaine, D. S. Lee, G. Myhre, M. Köhler, G. Pitari, U. Schumann, F. Stodal, C. Zerefos, 2005: Aviation radiative forcing in 2000: An update of IPCC (1999). -
Meteorol. Z. 14, 555-561.

SCHUMANN, U., 1996: On conditions for contrail formation. - Meteorol. Z. 5, 4-23.

Sharman, R., C. Tebaldi, G. Wiener, J. WolfF, 2005: An integrated approach to mid- and upper-level turbulence forecasting. - Wea. Forecast. 21, 268-287.

SMOlarkiewicz, P., L. MARGOLIN, 1997: On forward-intime differencing for fluids: an Eulerian/Semi-Lagrangian non-hydrostatic model for stratified flows. - Atmos.Oceans 35, 127-152.

-, 1998: MPDATA: A finite-difference solver for geophysical flows. - J. Comp. Phy. 140, 459-480.

SPICHTINGER, P., K. GieRENS,2008: Mesoscale modelling of homogeneous and heterogeneous cirrus cloud formation and evolution using EuLag. Part 1: Model description and validation. - Atmos. Chem. Phys. Diss. 8, 601-686.

Stuber N., P. Forster, G. RAdel, K. Shine, 2006: The importance of the diurnal and annual cycle of air traffic for contrail radiative forcing. - Nature 441, 864-867.

StröM, L., K. Gierens, 2002: First simulations of cryoplane contrails. - J. Geophys. Res. 107, 4346, doi:10.1029/2001JD000838.

SuSSMANN, R., K. GIERENS, 1999: Lidar and numerical studies on the different evolution of a contrail's vortex system and its secondary wake. - J. Geophys. Res. 104, 21312142.

,,-- 2001 : Differences in early contrail evolution of 2engined versus 4-engined aircraft. Lidar measurements and numerical simulations. - J. Geophys. Res. 106, 4899 4911. 